\title{
The Fermi Sky in a Multimessenger Context
}

\section{Felicia Krauß for the Fermi-LAT collaboration*}

GRAPPA \& Anton Pannekoek Institute for Astronomy, University of Amsterdam, Science Park 904, 1098 XH Amsterdam, The Netherlands

The Fermi Gamma-Ray Space Telescope has been launched on June 11 2008. While it has fundamentally changed our understanding of the high-energy $\gamma$-ray sky, it is even more powerful in multiwavelength and multimessenger efforts. In this work I summarize results from Fermi-LAT in the multimessenger context, pertaining to high-energy neutrinos.

Neutrino Oscillation Workshop

4 - 11 September, 2016

Otranto (Lecce, Italy)

${ }^{*}$ Speaker. 


\section{Cosmic rays}

Earth undergoes a constant influx of charged particles and nuclei, mostly protons. These cosmic rays have an $\sim E^{-2}$ spectrum with two 'breaks', at $\sim 10^{7}$ and $\sim 10^{9} \mathrm{GeV}$, called the knee and the ankle, respectively. Cosmic rays at energies up to the ankle are typically thought to originate in Galactic sources, including (but not limited to) supernovae, supernovae remnants and pulsar wind nebulae. Supernovae are currently the only confirmed extrasolar neutrino emitters [SN 1987A; for a review see 1] and therefore contribute to the low energy cosmic-ray spectrum [for a review see $2,3]$. As the cosmic-ray spectrum extends up to energies of $\sim 10^{21} \mathrm{eV}$, a long standing questions is: which sources can accelerate cosmic rays to these ultra-high energies? Direct identification of the origin of cosmic-ray protons and heavier nuclei is naturally hindered by magnetic fields: the Galactic magnetic field, Earth's magnetic field, and possibly intergalactic magnetic fields [IGMF; 4]. These deflect the charged particles.

\subsection{Photopion production}

If an astrophysical source is capable of accelerating protons to very high and ultra high energies a photon seed field is required at $\sim 10^{19} \mathrm{eV}$, below which proton-proton interactions dominate. At ultra-high energies the protons disintegrate in interactions with UV to X-ray photons resulting in charged and neutral pions. Neutral pions decay into a high-energy photon pair; this process is called photopion production. These photons can have energies between $\mathrm{keV}$ (X-ray) and $\mathrm{TeV}$ ( $\gamma$-rays), with an energy of $\sim 10 \%$ of the proton energy, providing a hadronic explanation for the highenergy emission that is seen in many Galactic and extragalactic astrophysical objects [5-7]. TeV $\gamma$ rays, however, likely do not escape the dense environments and produce an electron-positron pair. This makes high-energy photons in the $\mathrm{keV}$ to $\mathrm{GeV}$ energy range a powerful tool for identifying sources of cosmic rays. A detection of high-energy electromagnetic emission is not evidence for hadronic processes, however, as the high-energy emission of many sources can also be explained by the Inverse Compton process. Low energy photons (e.g., produced by Synchrotron emission of relativistic electrons or from an external photon field) are up-scattered by relativistic electrons. In many sources current leptonic and hadronic models can explain the available data equally well [e.g., 8]. In some sources a certain model is favored over another, but in general a broadband spectrum of a source does not guarantee to be able to distinguish between leptonic and hadronic scenarios.

\subsection{Expected neutrino emission}

In the hadronic case the charged pions decay into muons and neutrinos. The muons then further produce neutrinos. Assuming isospin symmetry, a correlation between the high-energy electromagnetic flux (assuming it stems from the decay of neutral pions) and high-energy neutrinos is expected with $F_{\gamma}=F_{v}$. An association of high-energy neutrinos with a high energy source would be unambiguous proof of the hadronic origin and contribute significantly to the knowledge of cosmic ray emitters. An association at a high statistical significance remains elusive but is within reach in the next few years with increasing detections of high-energy neutrino events. In order to understand the neutrino signal, it is crucial to have time-resolved information of the high-energy electromagnetic emission. 


\section{Fermi and the Large Area Telescope}

The Fermi Gamma-ray Space Telescope has two instruments on board, the Gamma-ray Burst Monitor (GBM) and the Large Area Telescope(LAT) [9]. It is an ideal instrument to study possible neutrino counterparts as it is continuously monitoring the sky, providing spectral and flux information for the full sky since its launch in 2008. The GBM monitors the sky at MeV energies in order to detect and study Gamma-ray Bursts (GRBs) and other transient events such as Terrestrial Gamma-ray Flashes [TGFs; 10]. The Large Area Telescope (LAT) operates at higher energies above $\sim 20 \mathrm{MeV}$. It is a pair conversion telescope with a combination of silicon microstrip detectors and tungsten conversion foils at the top of the instruments and a CsI scintillator calorimeter below. It observes the entire sky in three hours [9]. Its large effective area, angular resolution, energy range, and field of view make it an ideal instrument to also study faint extragalactic sources and search for counterparts of high-energy neutrinos. The largest fraction of sources it detects are blazars, a subclass of Active Galactic Nuclei (AGN) with a small angle between the line of sight and their relativistic outflows, called jets. Surprisingly, the second largest fraction of LAT sources [in the latest 3FGL catalog; 11] are unassociated. It is generally believed that these are largely blazars without known counterparts at other wavelengths, though it is unclear what makes them $\gamma$-ray bright, but faint at other energies.

\section{IceCube detection of astrophysical neutrinos}

Much progress has been made in the last few years concerning the detection of neutrinos, with the finalization of the IceCube detector at the South Pole in 2010, and the ANTARES experiment in the Mediterranean in 2008. IceCube has detected $\sim 50$ fully contained neutrino events, with a significant excess above the expected atmospheric background. The spectrum of atmospheric neutrinos is expected to fall steeply with increasing energy, with only very few events expected above $100 \mathrm{TeV}$ and none above $1 \mathrm{PeV}$. I therefore only consider those $\mathrm{PeV}$ neutrinos as the best possible way to identify astrophysical sources of cosmic rays [12-15]. Unfortunately the detection of high-energy neutrino events does not lead to a direct association with a Fermi-LAT counterpart, as these neutrino events often have large angular uncertainties $\left(\sim 10-15^{\circ}\right.$ for shower-like $v_{e}$ events, and $\sim 1^{\circ}$ for $v_{\mu}$ track events). Further detections of muon track events with small angular uncertainties offer a better possibility of a direct association. Additionally it has to be taken into account that a large number of sources remains unresolved by Fermi-LAT. We therefore do not expect an association for each event. Studying the Fermi-LAT extragalactic background makes it possible to estimate the fraction of known and unknown counterparts. We previously found that only $30 \%$ of neutrino events are expected to be associated with a LAT counterpart, assuming blazars as the sources of the neutrinos [16, 17].

\section{Astrophysical sources of the neutrino signal}

In this section I briefly summarize candidates for the observed neutrino emission and give the results that have come out of studies with Fermi-LAT data. 


\subsection{Galactic sources}

For most Galactic high-energy emitters neutrino production has been predicted. These include pulsars [18-23], X-ray and $\gamma$-ray binaries [24-30], supernovae and pulsar wind nebulae [31-36], and the Fermi bubbles [37-40]. The LAT found evidence for pion decay signatures in the spectrum of many supernova remnants [41-45]. While these likely largely contribute to the neutrino flux at lower energies it is theoretically difficult to explain neutrinos up to PeV energies, with the highest predicted energy at $300 \mathrm{TeV}$. It has further been argued that high-energy cosmic rays must originate from outside of the Galactic disk as their Larmor motion can not be contained [46, 47].

\subsection{Extragalactic sources}

The observed distribution of all contained neutrino events is consistent with being isotropic across the sky, indicating a strong extragalactic component. Possible extragalactic sources of cosmic rays and neutrinos are GRBs [48-53], blazars and non-blazar AGN [5, 6, 54, 55], and starburst galaxies [56, 57]. Starburst galaxies are also not able to produce $\mathrm{PeV}$ neutrino events $[56,58]$. AGN and GRBs sources are theoretically able to accelerate protons to sufficiently high energies to produce $\gtrsim 1 \mathrm{PeV}$ neutrinos.

\subsection{Gamma-ray Bursts}

Long GRBs can occur with type Ib/c supernovae [for a review see 59], though this is not the case for all long GRBs $[60,61]$. The dense iron core of the star undergoing a supernova collapses into a black hole. Further infalling material forms an accretion disk, which ejects relativistic jets. These travel outwards rapidly, and interact with the outer infalling layers. This produces lowenergy $\gamma$ rays. As this jet travels further outward it can interact (and be slowed) by the ambient medium which produces the afterglow at X-ray, optical and radio wavelengths. It is unclear whether protons are accelerated in this relativistic outflow and whether high-energy neutrinos are produced. It has been shown by [62] we only expect a low flux of neutrinos from GRBs, inconsistent with the observed PeV signal. LAT results confirm this and suggest that GRBs cannot be the dominant source of the IceCube neutrinos [63]. This is also in agreement with an All-Sky search by [64]. The GRB contribution could be higher for a large number of hypernovae [65]. No prompt neutrino event is currently consistent with a known GRB. Production of neutrinos at PeV-EeV energies is possible in GRB afterglows. As there currently is a lack of GRBs that are consistent with the observed neutrino events, chocked jets and low luminosity GRBs are being considered [65, 66]. Chocked jets are shortened by interaction with the surrounding medium. Weak jets would produce low luminosities. Either case would be very difficult to detect in the electromagnetic spectrum and this option cannot be ruled out currently.

\subsection{Active Galactic nuclei}

Neutrino emission has been predicted for the cores and jets of AGN. As a correlation between the high-energy EM flux and the neutrino flux is typically expected, it is likely that bright highenergy emitters contribute to the flux. Blazars are the dominant class seen by Fermi-LAT and the most luminous persistent sources. This makes them reasonable candidates for contributing to the flux of neutrinos. 
Blazars are typically further subdivided into two classes, BL Lacertae (BL Lacs) and flatspectrum radio quasars (FSRQs). Quasars tend to be more luminous than BL Lacs, with their spectra peaking at lower energies. Quasars also typically exhibit a strong thermal emission peak in the optical/UV, called the 'big blue bump' (BBB). This emission feature is generally interpreted to be the thermal emission from the accretion disk. And while several problems exist with this classification [see 67], the accretion disk would provide an adequate photon seed field for photopion production. BL Lac objects are often TeV emitter. In addition to the lack of a photon seed field, this makes it hard to explain the escape of $\mathrm{TeV}$ photons from the dense environments required for photopion production.

Several studies suggest that blazars alone cannot explain all IceCube contained neutrino events [68, 69]. This is not incompatible with the PeV events steming from AGN. [70] propose a combination of BL Lac events and PWN for all of the IC events in three years of data.

We used the six $\gamma$-ray and radio brightest blazars to estimate the integrated neutrino output. We found that the emission can be explained by these events, even without including contributions from fainter blazars or unresolved blazars [71]. None of the sources had a high individual expected neutrino output, which would explain the events. This work showed that blazars as a class are capable of producing the observed PeV events.

A follow-up study by ANTARES found two possible neutrino events for the two sources with the highest expected neutrino output and helped to constrain the spectral index of the neutrino spectrum [72].

For the third event at $\mathrm{PeV}$ energies, we found one blazar dominating the expected integrated neutrino output as it was undergoing a dramatic outburst (a state of high activity with a strongly increased flux). An increase in em flux is also expected to increase the neutrino flux. For small time scales this is typically negligible due to the low chance of detecting neutrinos. This was an outburst that lasted for a year, and only 8 outbursts reaching a similar fluence where detected over the lifetime of Fermi, with several of these before the start of the IceCube experiment. Unfortunately, we estimate that a $5 \%$ probability of a chance coincidence remains. It is an interesting result that will be be confirmed or refuted in the future. Assuming a physical connection between both, this would constrain the neutrino velocity to two orders of magnitude better than SN1987A [17].

The fourth event was detected at 2.6 PeV, with a low angular resolution [73]. Further events are now communicated on a short time scale via the GCN network and the Fermi LAT Galactic and Extragalactic Science Teams coordinated their efforts of searching for counterparts, but none was found $[74,75]$. It is worth noting that the $\gamma$-ray bright blazar PG $1553+113$ is located close to the position of the neutrino event.

\section{Conclusion}

High-energy neutrino events at $\gtrsim 1 \mathrm{PeV}$ are promising for identifying the sources of Ultra-high energy cosmic rays (UHECRs). Current LAT results suggest that AGN are the most likely candidates, although GRBs cannot be ruled out completely. It is reasonable to assume that several source classes contribute to the observed IceCube events. I find that blazars, as the most luminous, persistent, and numerous source class at high energies, are able to calorimetrically explain the observed IceCube events [71]. We further find one blazar in outburst, coincident with the third $\mathrm{PeV}$ event 
[17]. This blazar's outburst was one of the most dramatic outbursts at $\gamma$-ray wavelengths, but also at radio wavelengths, as observed by TANAMI. While this association is not statistically significant it is a tantalizing results. Only better statistics of the neutrino events will be able to confirm or refute the blazar hypothesis. It is further necessary to study the non-detection of neutrinos from other bright blazars is still in agreement with the hypothesis. It is possible that individual sources have different fractions of hadronic/leptonic high-energy emission production.

With the construction of the future Km3Net [76] array with a much larger volume and IceCube Gen-2 [77], a significant association of a neutrino event and an astrophysical source will be feasible. It is not unlikely that Fermi-LAT will still be operational at this point, providing crucial information about $\gamma$-ray bright sources. The planned Cherenkov Telescope Array [CTA; 78], which will detect $\gamma$-rays at $\mathrm{TeV}$ energies, will be useful in supporting Fermi-LAT observations and further constraining the spectral shape of $\gamma$-ray sources.

\section{Acknowledgments}

I thank D. Berge for the helpful comments. I acknowledges funding from the European Union's Horizon 2020 research and innovation programme under grant agreement No. 653477. The Fermi-LAT Collaboration acknowledges support for LAT development, operation and data analysis from NASA and DOE (United States), CEA/Irfu and IN2P3/CNRS (France), ASI and INFN (Italy), MEXT, KEK, and JAXA (Japan), and the K.A. Wallenberg Foundation, the Swedish Research Council and the National Space Board (Sweden). Science analysis support in the operations phase from INAF (Italy) and CNES (France) is also gratefully acknowledged.

\section{References}

[1] W. D. Arnett et al., ARA\&A 27, 629 (1989).

[2] P. Blasi, Astron. Astrophys. Rev. 21, 70 (2013).

[3] E. Amato, International Journal of Modern Physics D 23, 1430013 (2014).

[4] P. L. Biermann, Journal of Physics G Nuclear Physics 23, 1 (1997).

[5] K. Mannheim, A\&A 269, 67 (1993).

[6] K. Mannheim, Astropart. Phys. 3, 295 (1995a).

[7] K. Mannheim, A\&A 297, 321 (1995b).

[8] M. Böttcher et al., ApJ 768, 54 (2013).

[9] W. B. Atwood et al., ApJ 697, 1071 (2009).

[10] C. Meegan et al., ApJ 702, 791 (2009).

[11] F. Acero et al., ApJS 218, 23 (2015).

[12] M. G. Aartsen et al., Phys. Rev. L 111, 021103 (2013).

[13] IceCube Collaboration, Science 342, 1242856 (2013).

[14] M. G. Aartsen et al., Phys. Rev. L 113, 101101 (2014).

[15] The IceCube Collaboration, In: ICRC 2015, The Hague, The Netherlands, PoS(ICRC2015)1081

[16] F. Krauß et al., In: 5th Fermi Symposium, Nagoya, Japan, arXiv:1502.02147

[17] M. Kadler et al., Nature Physics 12, 807 (2016).

[18] D. Eichler, Nature 275, 725 (1978).

[19] A. K. Harding et al., ApJ 358, 561 (1990).

[20] L. A. Anchordoqui et al., ApJ 589, 481 (2003).

[21] B. Link et al., Physical Review Letters 94, 181101 (2005).

[22] B. Link et al., MNRAS 371, 375 (2006).

[23] A. Bhadra, International Cosmic Ray Conference 2, 775 (2008).

[24] T. K. Gaisser et al., Phys. Rev. L 54, 2265 (1985).

[25] D. Eichler et al., ApJ 402, 271 (1993).

[26] A. Levinson et al., Phys. Rev. L 87, 171101 (2001). 
[27] W. Bednarek, MNRAS 363, L46 (2005).

[28] S. Razzaque et al., Phys. Rev. D 82, 123012 (2010).

[29] J. Sitarek et al., Phys. Rev. D 86, 063011 (2012).

[30] W. Bednarek et al., Phys. Rev. D 90, 103008 (2014).

[31] K. S. Cheng et al., Journal of Physics G Nuclear Physics 16, 1115 (1990).

[32] F. A. Aharonian et al., A\&A 309, 917 (1996).

[33] R. J. Protheroe et al., Astroparticle Physics 9, 1 (1998).

[34] W. Bednarek et al., A\&A 405, 689 (2003).

[35] M. Mandelartz et al., Astroparticle Physics 65, 80 (2015).

[36] I. Di Palma et al., ApJ, submitted, arXiv:1605.01205 (2016).

[37] R. M. Crocker, MNRAS 423, 3512 (2012).

[38] C. Lunardini et al., Phys. Rev. L 108, 221102 (2012).

[39] S. Razzaque, Phys. Rev. D 88, 081302 (2013).

[40] C. Lunardini et al., Phys. Rev. D 92, 021301 (2015).

[41] A. A. Abdo et al., ApJL 706, L1 (2009).

[42] T. Tanaka et al., ApJL 740, L51 (2011).

[43] M. Ajello et al., ApJ 744, 80 (2012).

[44] F. Giordano et al., ApJL 744, L2 (2012).

[45] M. Ackermann et al., Science 339, 807 (2013).

[46] G. Cocconi, Il Nuovo Cimento (1955-1965) 3, 1433 (1956).

[47] G. Cocconi, Astroparticle Physics 4, 281 (1996).

[48] E. Waxman, Phys. Rev. L 75, 386 (1995).

[49] E. Waxman et al., Phys. Rev. L 78, 2292 (1997).

[50] E. Waxman et al., Phys. Rev. D 59, 023002 (1999).

[51] C. D. Dermer, ApJ 574, 65 (2002).

[52] C. D. Dermer et al., Phys. Rev. L 91, 071102 (2003).

[53] K. Murase et al., Phys. Rev. L 111, 121102 (2013).

[54] F. W. Stecker et al., Phys. Rev. L 66, 2697 (1991).

[55] K. Murase et al., Phys. Rev. D 90, 023007 (2014).

[56] A. Loeb et al., JCAP 5, 003 (2006).

[57] K. Murase et al., Phys. Rev. D 88, 121301 (2013).

[58] I. Tamborra et al., JCAP 9, 043 (2014).

[59] P. Mészáros, Reports on Progress in Physics 69, 2259 (2006).

[60] M. H. P. M. van Putten, MNRAS 396, L81 (2009).

[61] M. H. P. M. van Putten et al., MNRAS 444, L58 (2014).

[62] J. K. Becker et al., ApJ 721, 1891 (2010).

[63] K. Bechtol et al., ArXiv:1511.00688 (2015). submitted to ApJ

[64] M. G. Aartsen et al., ApJ 824, 115 (2016).

[65] N. Senno et al., Phys. Rev. D 93, 083003 (2016).

[66] K. Murase et al., Physical Review Letters 116, 071101 (2016).

[67] A. Lawrence, MNRAS 423, 451 (2012).

[68] M. R. Feyereisen et al., ArXiv:1610.01607 (2016).

[69] IceCube Collaboration et al., ArXiv:1611.03874 (2016a).

[70] P. Padovani et al., MNRAS 443, 474 (2014).

[71] F. Krauß et al., A\&A 566, L7 (2014).

[72] ANTARES Collaboration et al., A\&A 576, L8 (2015).

[73] IceCube Collaboration et al., ArXiv:1607.08006 (2016b).

[74] G. Vianello et al., GRB Coordinates Network 19360 (2016a).

[75] G. Vianello et al., The Astronomer's Telegram 9008 (2016b).

[76] S. Adrián-Martínez et al., Journal of Physics G Nuclear Physics 43, 084001 (2016).

[77] IceCube-Gen2 Collaboration et al., arXiv:1412.5106 (2014).

[78] B. S. Acharya et al., Astroparticle Physics 43, 3 (2013).

[79] A. Mücke et al., Nuclear Physics B Proceedings Supplements 80, C810 (2000).

[80] The IceCube-Gen2 Collaboration et al., In: 34th ICRC, The Hague 2015, PoS(ICRC2015) 